\title{
An Observation of the Hematological Profile and Histopathological Findings in Patients with Cervical Lymphadenopathy
}

\author{
Dr. Shaha Md. Ashrafozzaman ${ }^{1 *}$, Professor Md. Robed Amin², Dr. Hasina Akhtar
}

\author{
1Assistant Professor, Department of Medicine, Mymensingh Medical College Hospital, Mymensingh, Bangladesh \\ 2Professor, Department of Medicine, Dhaka Medical College, Dhaka, Bangladesh \\ 3Lecturer, Department of Pharmacology, Mymensingh Medical College, Mymensingh, Bangladesh
}

DOI: $10.36347 /$ sjams.2020.v08i11.046

| Received: 12.10.2020 | Accepted: 26.10.2020 | Published: 30.11.2020

*Corresponding author: Dr. Shaha Md. Ashrafozzaman

Abstract

Original Research Article

This cross sectional observational study was carried out with an aim to describe the types of variations in clinical presentation, demographic characteristics, hematological profile \& histological findings and etiological pattern of patient having cervical lymphadenopathy. A total of 115 patients presented with cervical lymphadenopathy both inpatient and outpatient department of Medicine in Dhaka Medical College Hospital, during March 2014 to December 2014, were included in this study. A total of 115 consecutive patients having cervical lymphadenopathy both inpatient and outpatient department in the above mentioned hospitals were enrolled in this study to see the clinic-pathological correlation. Almost one third (29.6\%) patients belonged to age 21-30 years and the mean age was found $42.1 \pm 15.6$ years. The association between age with histological findings it was observed that lymphoma was found $27.6 \%$ in patients belonged to age 41-50 years, tuberculosis 14(43.8\%) patients belonged to age 21-30 years, metastatic carcinoma was $10(41.7 \%)$ patients belonged to age 51-60 years, nonspecific lymphadenopathy $11(45.8 \%)$ patients belonged to age 21-30 years. Male to female ratio was 2.6:1. More than three fourth patients were married. More than one fourth, patients were laborer, one third were businessman, and others were housewives, student, and unemployed. More than half of the patients came from lower class and others from middle class family.

Keywords: Clinico-pathological, Characteristics, Hematological, Histological, ymphadenopathy. Copyright $\left({ }^{\circ} 2020\right.$ The Author(s): This is an open-access article distributed under the terms of the Creative Commons Attribution 4.0 International License (CC BY-NC 4.0) which permits unrestricted use, distribution, and reproduction in any medium for non-commercial use provided the original author and source are credited.

\section{INRTODUCTION}

In Bangladesh a cross sectional study conducted in the department of Pathology, Enam Medical College \& Hospital, Savar, Dhaka during the period from January 2006 to December 2010. A total of 107 patients were evaluated for specific cause of cervical lymphadenopathy and found $54.2 \%$ were males and $45.8 \%$ females. The age of the patients ranged from 2 to 85 years with a mean age of $32.68 \pm 18.01$ years. Of the 107 lymph node biopsies, $31.8 \%$ were reactive lymphadenitis, $38.3 \%$ tuberculosis, $1.9 \%$ was noncaseous granuloma, 5.6\% Hodgkin lymphoma, $7.5 \%$ non-Hodgkin lymphoma, $11.2 \%$ metastatic neoplasm and $3.7 \%$ were other specific lesions. The commonest cause of cervical lymphadenopathy was tuberculosis, followed by reactive lymphadenitis, lymphoma and metastatic neoplasm. A BCPS dissertation shows out of 50 cases, biopsies were done in 31 cases which revealed lymphoma in 14, tuberculosis in 10, metastatic carcinoma in 5, and non-specific finding in 2 cases. FNAC were done in 7 cases out of which shows metastatic carcinoma in 4 , non-specific findings 2 , and tuberculosis in 1 case [1]. In everyday practice cervical lymphadenopathy is a common finding in a large proportion of patients. Most patients can be diagnosed on the basis of careful history, physical examination and investigations. Considering the previous studies done abroad among different ethnic area it can be said that there is great variation in etiology of cervical lymphadenopathy along with the clinical presentation. This study is intended to be performed to evaluate the common and uncommon etiology on the basis of histopathology as well as identify any non-specific cervical lymphadenopathy in Bangladeshi adult population. Cervical lymph node enlargement is common clinical finding in medical practice. Enlargement of lymph node may result from proliferation of lymphocytes intrinsic to the lymph node either due to infection or due to lymphoproliferative disorder or from the migration $\&$ infiltration of nodal tissue by either extrinsic inflammatory cells or metastatic malignant cells [2]. Cervical lymphadenopathy may be due to Infections, Malignancy, Autoimmune diseases, Miscellaneous/unusual conditions, and iatrogenic 
causes [3]. Any failure to decrease in size of lymph node within 10-14 days of treatment, a need for further evaluation is indicated $[4,5]$. The most common cause of cervical lymphadenopathy is reactive hyperplasia resulting from an infectious process, most commonly a viral upper respiratory tract infection [6]. Upper respiratory tract infection might be caused by rhinovirus, Parainfluenza virus, influenza virus, respiratory syncytial virus, coronavirus, adenovirus, or reovirus. Other viruses associated with cervical lymphadenopathy include Epstein-Barr virus (EBV), cytomegalovirus, rubella, rubeola, varicella-zoster virus, herpes simplex virus (HSV), coxsackievirus, and human immunodeficiency virus (HIV). Bacterial cervical lymphadenitis is usually caused by group A $\beta$ hemolytic streptococci or Staphylococcus aureus. Anaerobic bacteria can cause cervical lymphadenitis, usually in association with dental caries and periodontal disease. Group B streptococci and Haemophilus influenzae type $b$ are less frequent causal organisms. Diphtheria is a rare cause. Bartonella henselae (cat scratch disease), atypical mycobacteria, and mycobacteria are important causes of subacute or chronic cervical lymphadenopathy [7]. Chronic posterior cervical lymphadenitis is the most common form of acquired toxoplasmosis and is the sole presenting symptom in 50\% of cases [8]. In 2006 Yaris et al., performed a retrospective review of 126 patients in USA. Of the 126 patients $22.2 \%$ were found to have disease other than lymphadenopathy. Of those with lymphadenopathy, $76.6 \%$ had benign disease mostly belonging to acute lymphadenitis and $23.4 \%$ had malignancies. In a study performed by Ellison et al., [9] in 1999 of 309 clavicular fine needle aspirations, they found that $55 \%$ of nodes sampled were malignant. Zeharia et al., [10] performed retrospectively on 92 children diagnosed with atypical mycobacterial lymphadenopathy. The parents of all 92 children in this study opted for non-surgical and non-medical conservative management, and patients were followed for a minimum of 2 years. Clinical profile includes $80 \%$ of patients were less than 4 years old, $80 \%$ of patients had lymphadenopathy greater than $3 \mathrm{~cm}$ in size, $90 \%$ of patients had unifocal lymphadenopathy, Lymphadenopathy was most commonly found in Submandibular (50\%), Cervical (25\%), Pre-auricular $(10 \%)$ regions, $85 \%$ of patients had a positive PPD $(>10 \mathrm{~mm}), 90 \%$ of cases were due to M. aviumintracellulare and M. haemophilum, $97.4 \%$ of patients had a dominant node with purulent drainage for 3-8 weeks. To observe the hematological profile \& histopathological findings in these patients.

\section{OBJECTIVES \\ General Objective}

- To observe the hematological profile \& histopathological findings in patients with cervical lymphadenopathy

\section{Specific Objectives}

- To describe the types of variations in clinical presentation of cervical lymphadenopathy.

- To find out the etiological pattern of cervical lymphadenopathy.

- To describe the demographic characteristics of patient having cervical lymphadenopathy.

\section{Mrthodology and Materials}

This cross sectional observational study was carried out with an aim to describe the types of variations in clinical presentation, demographic characteristics, hematological profile \& histological findings and etiological pattern of patient having cervical lymphadenopathy. A total of 115 patients presented with cervical lymphadenopathy both inpatient and outpatient department of Medicine in Dhaka Medical College Hospital, during March 2014 to December 2014, were included in this study. Patients aged $\geq 18$ years, presented with cervical lymphadenopathy $>1.0 \mathrm{~cm}$ in diameter, duration of cervical lymphadenopathy more than 14 days (subjective and objective) were enrolled in this study. Patients aged less than 18 years, extremely debilitated patient, patient with insignificant lymph node enlargement, e.g. $<1.0 \mathrm{~cm}$ in cervical regions, patients who refuse to give consent, known case of lymphadenopathy and patients suggestive of haematologic malignancy (leukaemia) were excluded from the study. The present study findings were discussed and compared with previously published relevant studies. The result of present study is as follows.

\section{Inclusion Criteria}

1. Patients aged 18 years and above.

2. Patients having cervical lymphadenopathy $>1.0 \mathrm{~cm}$ in diameter.

3. Patients presented in outdoor and indoor, department of medicine, Dhaka Medical College and Hospital.

4. Duration of cervical lymphadenopathy $\geq 14$ days (subjective and objective).

\section{Exclusion Criteria}

1. Patient with insignificant lymph node enlargement, e.g. $<1.0 \mathrm{~cm}$ in cervical regions.

2. The patients who refuse to give consent.

3. A diagnosed case of lymphadenopathy.

4. In those patient where FNAC/ biopsy is contraindicated, such as leukaemia.

\section{RESUlTS}

A total of 115 consecutive patients having cervical lymphadenopathy both inpatient and outpatient department in the above mentioned hospitals were enrolled in this study to see the clinicopathological correlation. Almost one third (29.6\%) patients belonged 
to age 21-30 years and the mean age was found $42.1 \pm 15.6$ years. The association between age with histological findings it was observed that lymphoma was found $8(27.6 \%$ )in patients belonged to age 41-50 years, tuberculosis $14(43.8 \%)$ patients belonged to age 21-30 years, metastatic carcinoma was $10(41.7 \%)$ patients belonged to age 51-60 years, nonspecific lymphadenopathy $11(45.8 \%)$ patients belonged to age 21-30 years. Male to female ratio was $2.6: 1$. More than three fourth patients were married. More than one fourth, patients were laborer, one third were businessman, and others were housewives, student, and unemployed. More than half of the patients came from lower class and others from middle class family. Regarding the clinical feature of the study patients, it was observed that majority patients had nonspecific symptom like generalized weakness, loss of appetite and weight loss, other features like fever, cough and headache also present in less number. Examination cervical lymph node observed that most of the lymph node was non tender, discreet in character, firm in consistency and mobile. On the other hand, lymph nodes fixed in metastatic carcinoma and matted in tuberculosis. On systemic examination, it was observed that splenomegaly and hepatomegaly were the common finding followed by ascites. Organomegaly was frequently seen in lymphoma while ascites in metastatic cases. More than three fourth of the patients had normal findings in CXR P/A view followed by features of consolidation, features of effusion, bilateral hilar shadow and features of fibrosis. Hemoglobin level showed most patients were anaemic. Erythrocyte sedimentation rate were also high in most of the patients. Total count of WBC of the study patients were within normal limit. FNAC of lymph node was done in 45 cases and among them tuberculosis was found $21(46.7 \%)$, lymphoma $8(17.8 \%)$, metastatic carcinoma $8(17.8 \%)$, nonspecific lymphadenopathy $6(13.3 \%)$ and others were $2(4.4 \%)$. Biopsy of lymph node was done in 70 cases among them 21(30.0\%) patients had lymphoma, $\quad 18(25.7 \%)$ had nonspecific lymphadenopathy, 16(22.9\%) had metastatic carcinoma, $11(15.7 \%)$ had tuberculosis. Diagnosis of study patients by histopathology and other relevant investigations finally observed that $34(29.6 \%)$ patients had tuberculosis, 29(25.2\%) had lymphoma, 24(20.9\%) had metastatic carcinoma, 14(12.2\%) had nonspecific lymphadenopathy.

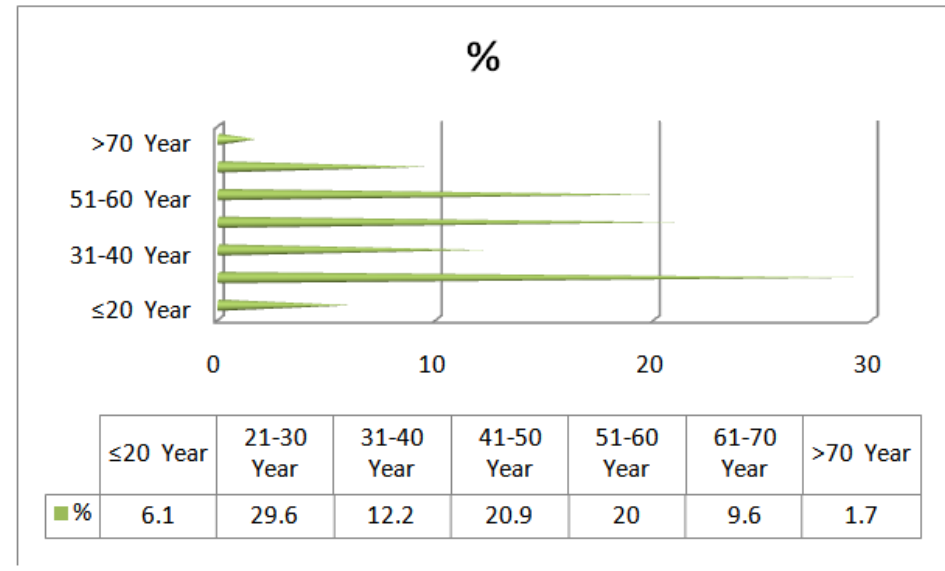

Fig-1: Distribution of study population by age $(n=115)$ Mean \pm SD: 42.1 \pm 15.6 , Range: $19-80$

Table-1: Distribution of study population by age ( $n=115)$

\begin{tabular}{|l|l|l|}
\hline Age (Years) & Number of patients & Percentage \\
\hline$\leq \mathbf{2 0}$ & 7 & 6.1 \\
\hline $\mathbf{2 1 - 3 0}$ & 34 & 29.6 \\
\hline $\mathbf{3 1 - 4 0}$ & 14 & 12.2 \\
\hline $\mathbf{4 1 - 5 0}$ & 24 & 20.9 \\
\hline $\mathbf{5 1 - 6 0}$ & 23 & 20.0 \\
\hline $\mathbf{6 1 - 7 0}$ & 11 & 9.6 \\
\hline $\mathbf{> 7 0}$ & 2 & 1.7 \\
\hline Mean \pm SD & 42.1 & \pm 15.6 \\
\hline Range (Min,max) & 19 &, 80 \\
\hline
\end{tabular}

Table shows age distribution of the study patients; it was observed that almost one third (29.6\%) patients belonged to age 21-30 years. The mean age was found $42.1 \pm 15.6$ years with range from 19 to 80 years. 
Table-2: Distribution of study population by age and histological findings ( $n=115)$

\begin{tabular}{|c|c|c|c|c|c|c|c|c|c|c|}
\hline \multirow[t]{3}{*}{ Age (Years) } & \multicolumn{10}{|c|}{ Histopathology Report } \\
\hline & \multicolumn{2}{|c|}{$\begin{array}{l}\text { Lymphoma } \\
(\mathbf{n}=29)\end{array}$} & \multicolumn{2}{|c|}{$\begin{array}{l}\text { Tuberculosis } \\
(\mathrm{n}=32)\end{array}$} & \multicolumn{2}{|c|}{$\begin{array}{l}\text { Metastatic Carcinoma } \\
(\mathrm{n}=\mathbf{2 4})\end{array}$} & \multicolumn{2}{|c|}{$\begin{array}{l}\text { Nonspecific lymphadenopathy } \\
(n=24)\end{array}$} & \multicolumn{2}{|c|}{$\begin{array}{l}\text { Others } \\
(n=6)\end{array}$} \\
\hline & $\mathrm{n}$ & $\%$ & $\mathrm{n}$ & $\%$ & $\mathrm{n}$ & $\%$ & $\mathrm{n}$ & $\%$ & $\mathrm{n}$ & $\%$ \\
\hline$<20$ & 2 & 6.9 & 2 & 6.3 & 0 & 0.0 & 2 & 8.3 & 1 & 16.7 \\
\hline $21-30$ & 4 & 13.8 & 14 & 43.8 & 3 & 12.5 & 11 & 45.8 & 2 & 33.3 \\
\hline $31-40$ & 5 & 17.2 & 6 & 18.8 & 0 & 0.0 & 1 & 4.2 & 2 & 33.3 \\
\hline 41-50 & 8 & 27.6 & 4 & 12.5 & 6 & 25.0 & 6 & 25.0 & 0 & 0.0 \\
\hline 51-60 & 6 & 20.7 & 3 & 9.4 & 10 & 441.7 & 4 & 16.7 & 0 & 0.0 \\
\hline $61-70$ & 4 & 13.8 & 2 & 6.3 & 4 & 16.7 & 0 & 0.0 & 1 & 16.7 \\
\hline$>70$ & 0 & 0.0 & 1 & 3.1 & 1 & 4.2 & 0 & 0.0 & 0 & 0.0 \\
\hline
\end{tabular}

Table shows age and histological findings of the patients. It was observed that in lymphoma majority $8(27.6 \%)$ patients belonged to age group of $41-50$ years, in tuberculosis $14(43.8 \%)$ patients belonged to age group of 21-30 years, in metastatic carcinoma was $10(41.7 \%)$ patients belonged to age group of 51-60 years, in nonspecific lymphadenopathy 11(45.8\%) patients belonged to age group of 21-30 years

Table-3: Distribution of study population by sex with marital status $(n=115)$

\begin{tabular}{|l|l|l|l|l|l|}
\hline Sex & n & \multicolumn{2}{|c|}{ Married } & \multicolumn{2}{l|}{ Unmarried } \\
\hline & & $\mathrm{n}$ & $\%$ & $\mathrm{n}$ & $\%$ \\
\hline Male & 83 & 65 & 78.3 & 18 & 21.7 \\
\hline Female & 32 & 24 & 75.0 & 8 & 25.0 \\
\hline Total & 115 & 89 & 77.4 & 26 & 22.6 \\
\hline
\end{tabular}

Table shows sex of the study patients, it was observed that $83(72.2 \%)$ patients were male, among them $65(78.3 \%)$ patients were married and $18(21.7 \%)$ were unmarried. Thirty two $(28.8 \%)$ patients were female, among them $24(75.0 \%)$ patients were married and $8(25.0 \%)$ were unmarried.

Table-4: Distribution of positive clinical symptoms by histopathological findings (n=115)

\begin{tabular}{|c|c|c|c|c|c|c|c|c|c|c|c|}
\hline \multirow{3}{*}{$\begin{array}{ll}\text { Positive } & \text { clinical } \\
\text { symptoms } & \end{array}$} & \multicolumn{10}{|c|}{ Histopathological findings } & \multirow{3}{*}{$\begin{array}{l}P \\
\text { value }\end{array}$} \\
\hline & \multicolumn{2}{|c|}{$\begin{array}{l}\text { Lymphoma } \\
(n=29)\end{array}$} & \multicolumn{2}{|c|}{$\begin{array}{l}\text { Tuberculosis } \\
(\mathbf{n}=32)\end{array}$} & \multicolumn{2}{|c|}{$\begin{array}{l}\text { Metastatic } \\
\text { carcinoma } \\
(n=24)\end{array}$} & \multicolumn{2}{|c|}{$\begin{array}{l}\text { Nonspecific } \\
\text { lymphadenopathy } \\
(\mathrm{n}=24)\end{array}$} & \multicolumn{2}{|c|}{$\begin{array}{l}\text { *Other } \\
(\mathrm{n}=6)\end{array}$} & \\
\hline & $\mathbf{n}$ & $\%$ & $\mathbf{n}$ & $\%$ & $\mathbf{n}$ & $\%$ & $\mathbf{n}$ & $\%$ & $\mathbf{n}$ & $\%$ & \\
\hline \multicolumn{12}{|l|}{ Generalized weakness } \\
\hline Present & 28 & 96.6 & 31 & 96.9 & 23 & 95.8 & 21 & 87.5 & 2 & 33.3 & \multirow[t]{2}{*}{0.001} \\
\hline Absent & 1 & 3.4 & 1 & 3.1 & 1 & 4.2 & 3 & 12.5 & 4 & 66.7 & \\
\hline \multicolumn{12}{|l|}{ Appetite } \\
\hline Present & 1 & 3.4 & 0 & 0.0 & 0 & 0.0 & 15 & 62.5 & 1 & 16.7 & \multirow[t]{2}{*}{$0.001^{\mathrm{s}}$} \\
\hline Lost & 28 & 96.6 & 32 & 100.0 & 24 & 100.0 & 9 & 37.5 & 5 & 83.3 & \\
\hline \multicolumn{12}{|l|}{ Weight loss } \\
\hline Present & 29 & 100.0 & 30 & 93.8 & 22 & 91.7 & 19 & 79.2 & 2 & 33.3 & \multirow[t]{2}{*}{$0.001^{\mathrm{s}}$} \\
\hline Absent & 0 & 0.0 & 2 & 6.3 & 2 & 8.3 & 5 & 20.8 & 4 & 66.7 & \\
\hline \multicolumn{12}{|l|}{ Cough } \\
\hline Present & 8 & 27.6 & 14 & 43.8 & 9 & 37.5 & 5 & 20.8 & 1 & 16.7 & \multirow{2}{*}{$\begin{array}{l}0.327^{\mathrm{n}} \\
\mathrm{s}\end{array}$} \\
\hline Absent & 21 & 72.4 & 18 & 56.3 & 15 & 62.5 & 19 & 79.2 & 5 & 83.3 & \\
\hline \multicolumn{12}{|l|}{ Sputum } \\
\hline Present & 3 & 37.5 & 13 & 92.9 & 6 & 66.7 & 4 & 80.0 & 0 & 0.0 & \multirow[t]{2}{*}{$0.037^{\mathrm{s}}$} \\
\hline Absent & 5 & 62.5 & 1 & 7.1 & 3 & 33.3 & 1 & 20.0 & 1 & 100.0 & \\
\hline \multicolumn{12}{|l|}{ Alteration of bowel habit } \\
\hline Present & 0 & 0.0 & 0 & 0.0 & 9 & 37.5 & 2 & 8.3 & 0 & 0.0 & \multirow[t]{2}{*}{$0.001^{\mathrm{s}}$} \\
\hline Absent & 29 & 100.0 & 32 & 100.0 & 15 & 62.5 & 22 & 91.7 & 6 & 100.0 & \\
\hline \multicolumn{12}{|l|}{ Haemoptysis } \\
\hline Present & 0 & 0.0 & 3 & 9.4 & 2 & 8.3 & 0 & 0.0 & 0 & 0.0 & \multirow{2}{*}{$\begin{array}{l}0.235^{\mathrm{n}} \\
\mathrm{s}\end{array}$} \\
\hline Absent & 29 & 100.0 & 29 & 90.6 & 22 & 91.7 & 24 & 100.0 & 6 & 100.0 & \\
\hline
\end{tabular}

$\mathbf{s}=$ significant, $\mathbf{n s}=$ not significant, $P$ value reached from chi square test

Generalized weakness was found commonly in lymphoma $(96.6 \%)$, tuberculosis $(96.9 \%)$, metastatic carcinoma $(95.8 \%)$ and in nonspecific lymphadenopathy $(87.5 \%)$. Appetite was lost in most cases except in nonspecific lymphadenopathy cases $(37.5 \%)$. Weight loss also present in most of the groups except group that includes others etiology. Sputum was most commonly found in tuberculosis $(92.9 \%)$, then in 
metastatic carcinoma (66.7\%) and less commonly in lymphoma and nonspecific lymphadenopathy. Alteration of bowel habit was present in metastatic carcinoma $(37.5 \%)$. Which is statistically significant $(<0.05)$.

Table-5: Distribution character of fever by histopathological findings $(n=115)$

\begin{tabular}{|c|c|c|c|c|c|c|c|c|c|c|c|}
\hline \multirow[t]{3}{*}{ Character of fever } & \multicolumn{10}{|c|}{ Histopathological findings } & \multirow{3}{*}{$\begin{array}{l}P \\
\text { value }\end{array}$} \\
\hline & \multicolumn{2}{|c|}{$\begin{array}{l}\text { Lymphoma } \\
(\mathbf{n}=29)\end{array}$} & \multicolumn{2}{|c|}{$\begin{array}{l}\text { Tuberculosis } \\
(n=32)\end{array}$} & \multicolumn{2}{|c|}{$\begin{array}{l}\text { Metastatic carcinoma } \\
(\mathrm{n}=24)\end{array}$} & \multicolumn{2}{|c|}{$\begin{array}{l}\text { Nonspecific lymphadenopathy } \\
(\mathrm{n}=24)\end{array}$} & \multicolumn{2}{|c|}{$\begin{array}{l}\text { Other } \\
(n=6)\end{array}$} & \\
\hline & n & $\%$ & n & $\%$ & $\mathbf{n}$ & $\%$ & n & $\%$ & n & $\%$ & \\
\hline \multicolumn{12}{|l|}{ Presence of fever } \\
\hline Present & 26 & 89.7 & 32 & 100.0 & 8 & 33.3 & 19 & 79.2 & 5 & 83.3 & \multirow[t]{2}{*}{$0.001^{\mathrm{s}}$} \\
\hline Absent & 3 & 10.3 & 0 & 0.0 & 16 & 66.7 & 5 & 20.8 & 1 & 16.7 & \\
\hline Grade of fever & \multicolumn{2}{|c|}{$(n=26)$} & \multicolumn{2}{|c|}{$(n=32)$} & \multicolumn{2}{|c|}{$(n=8)$} & \multicolumn{2}{|c|}{$(n=19)$} & \multicolumn{2}{|c|}{$(\mathrm{n}=5)$} & \\
\hline High grade & 7 & 26.9 & 5 & 15.6 & 7 & 87.5 & 5 & 26.3 & 3 & 60.0 & \multirow[t]{2}{*}{$0.001^{\mathrm{s}}$} \\
\hline Low grade & 19 & 73.1 & 27 & 84.4 & 1 & 12.5 & 14 & 73.7 & 2 & 40.0 & \\
\hline \multicolumn{12}{|l|}{$\begin{array}{l}\text { Associated } \\
\text { with sweating }\end{array}$} \\
\hline Present & 18 & 69.2 & 31 & 96.9 & 4 & 50.0 & 7 & 36.8 & 1 & 20.0 & \multirow[t]{2}{*}{$0.001^{\mathrm{s}}$} \\
\hline Absent & 8 & 30.8 & 1 & 3.1 & 4 & 50.0 & 12 & 63.2 & 4 & 80.0 & \\
\hline
\end{tabular}

$\mathrm{s}=$ significant, $\mathrm{P}$ value reached from chi square test.

Fever was found mainly in lymphoma $(89.7 \%)$, tuberculosis $(100.0 \%)$, nonspecific lymphadenopathy $(79.2 \%)$ and others $(83.3 \%)$ patient group findings. low grade fever was the dominant finding in lymphoma $(73.1 \%)$, tuberculosis $(84.4 \%)$ and nonspecific lymphadenopathy $(73.7 \%)$. High grade of fever was found in metastatic carcinoma (87.5\%). Fever associated with sweating was found mainly in lymphoma (69.2\%).

Table-6: Distribution of fever by histopathological findings $(n=90)$

\begin{tabular}{|c|c|c|c|c|c|c|c|c|c|c|c|}
\hline \multirow{3}{*}{$\begin{array}{l}\text { Duration of fever } \\
\text { (day) }\end{array}$} & \multicolumn{10}{|c|}{ Histopathological findings } & \multirow[t]{3}{*}{$P$ value } \\
\hline & \multicolumn{2}{|c|}{$\begin{array}{l}\text { Lymphoma } \\
(n=26)\end{array}$} & \multicolumn{2}{|c|}{$\begin{array}{l}\text { Tuberculosis } \\
(n=32)\end{array}$} & \multicolumn{2}{|c|}{$\begin{array}{l}\text { Metastatic } \\
\text { carcinoma } \\
(\mathrm{n}=8)\end{array}$} & \multicolumn{2}{|c|}{$\begin{array}{l}\text { Nonspecific } \\
\text { lymphadenopathy } \\
(\mathrm{n}=19)\end{array}$} & \multicolumn{2}{|c|}{$\begin{array}{l}\text { Other } \\
(n=5)\end{array}$} & \\
\hline & $\mathrm{n}$ & $\%$ & n & $\%$ & $\mathrm{n}$ & $\%$ & n & $\%$ & $\mathrm{n}$ & $\%$ & \\
\hline$<30$ & 1 & 3.9 & 2 & 6.3 & 4 & 50.0 & 1 & 5.2 & 4 & 80.0 & \\
\hline $30-60$ & 22 & 84.6 & 25 & 78.1 & 4 & 50.0 & 9 & 47.4 & 0 & 0.0 & \\
\hline$>60$ & 3 & 11.5 & 5 & 15.6 & 0 & 0.0 & 9 & 47.4 & 1 & 20.0 & \\
\hline Mean \pm SD & 53.3 & \pm 21.4 & 52.8 & \pm 27.3 & 26.4 & \pm 9.6 & 81.3 & \pm 54.9 & 32.8 & \pm 32.3 & $0.001^{\mathrm{s}}$ \\
\hline Range (min, max) & 20 &, 120 & 20 &, 150 & 14 & ,45 & 14 &, 180 & 14 & 90 & \\
\hline
\end{tabular}

$\mathrm{s}=$ significant, $\mathrm{P}$ value reached from ANOVA test.

Mean duration of fever was found $53.5 \pm 21.4$ days in lymphoma, $52.8 \pm 27.3$ days in tuberculosis, $26.4 \pm 9.6$ days in metastatic carcinoma, $81.3 \pm 54.9$ days in nonspecific lymphadenopathy and $32.8 \pm 32.3$ days in others histopathological findings. Which was statistically significant $(<0.05)$ among five groups.

Table-7: Distribution of lymph node character by histopathological findings $(n=115)$

\begin{tabular}{|c|c|c|c|c|c|c|c|c|c|c|c|}
\hline \multirow{3}{*}{$\begin{array}{l}\text { Lymph node } \\
\text { character }\end{array}$} & \multicolumn{10}{|c|}{ Histopathological findings } & \multirow[t]{3}{*}{$P$ value } \\
\hline & \multicolumn{2}{|c|}{$\begin{array}{l}\text { Lymphoma } \\
(n=29)\end{array}$} & \multicolumn{2}{|c|}{$\begin{array}{l}\text { Tuberculosis } \\
(\mathbf{n}=32)\end{array}$} & \multicolumn{2}{|c|}{$\begin{array}{l}\text { Metastatic } \\
\text { carcinoma } \\
(\mathrm{n}=24)\end{array}$} & \multicolumn{2}{|c|}{$\begin{array}{l}\text { Nonspecific } \\
\text { lymphadenopathy } \\
(\mathbf{n = 2 4 )}\end{array}$} & \multicolumn{2}{|c|}{$\begin{array}{l}\text { Other } \\
(n=6)\end{array}$} & \\
\hline & $\mathbf{n}$ & $\%$ & $\mathbf{n}$ & $\%$ & $\mathbf{n}$ & $\%$ & $\mathbf{n}$ & $\%$ & $\mathbf{n}$ & $\%$ & \\
\hline \multicolumn{12}{|l|}{ Tenderness } \\
\hline Preset & 1 & 3.4 & 2 & 6.3 & 2 & 8.3 & 0 & 0.0 & 5 & 83.3 & \multirow[t]{2}{*}{$0.001^{\mathrm{s}}$} \\
\hline Absent & 28 & 96.6 & 30 & 93.8 & 22 & 91.7 & 24 & 100.0 & 1 & 16.7 & \\
\hline \multicolumn{12}{|l|}{ Character } \\
\hline Matted & 1 & 3.4 & 11 & 34.4 & 1 & 4.2 & 1 & 4.2 & 0 & 0.0 & \multirow[t]{2}{*}{$0.001^{\mathrm{s}}$} \\
\hline Discreet & 28 & 96.6 & 21 & 65.6 & 23 & 95.8 & 23 & 95.8 & 6 & 100.0 & \\
\hline \multicolumn{12}{|l|}{ Consistency } \\
\hline Hard & 1 & 3.4 & 0 & 0.0 & 18 & 75.0 & 0 & 0.0 & 0 & 0.0 & \multirow[t]{2}{*}{$0.001^{\mathrm{s}}$} \\
\hline Firm & 28 & 96.6 & 32 & 100.0 & 6 & 25.0 & 24 & 100.0 & 6 & 100.0 & \\
\hline \multicolumn{12}{|l|}{ Fixity } \\
\hline Mobile & 28 & 96.6 & 32 & 100.0 & 7 & 29.2 & 24 & 100.0 & 5 & 83.3 & \multirow[t]{2}{*}{$0.001^{\mathrm{s}}$} \\
\hline Fixed & 1 & 3.4 & 0 & 0.0 & 17 & 70.8 & 0 & 0.0 & 1 & 16.7 & \\
\hline
\end{tabular}

$\mathrm{S}=$ significant, $\mathrm{P}$ value reached from chi square test 
Table Shows tenderness was found mostly in group that include others $(83.3 \%)$ histopathological finding followed by metastatic carcinoma $(8.3 \%)$ but in most other group's lymph node was non-tender. Matted character was found in tuberculosis $(34.4 \%)$, in most other cases lymph node was discreet. Hard in consistency mainly found in metastatic carcinoma (75.0\%) but most other group's lymph node was firm. Fixity to underlying structure was mainly found in metastatic carcinoma (70.8\%), most other cases lymphnode were mobile. Which is statistically significant $(<0.05)$.

Table-8: Distribution of positive sign by histopathological findings $(n=115)$

\begin{tabular}{|c|c|c|c|c|c|c|c|c|c|c|c|}
\hline \multirow[t]{3}{*}{ Positive sign } & \multicolumn{10}{|c|}{ Histopathological findings } & \multirow[t]{3}{*}{$P$ value } \\
\hline & \multicolumn{2}{|c|}{$\begin{array}{l}\text { Lymphoma } \\
(\mathrm{n}=29)\end{array}$} & \multicolumn{2}{|c|}{$\begin{array}{l}\text { Tuberculosis } \\
(\mathrm{n}=32)\end{array}$} & \multicolumn{2}{|c|}{$\begin{array}{l}\text { Metastatic carcinoma } \\
(\mathrm{n}=24)\end{array}$} & \multicolumn{2}{|c|}{$\begin{array}{l}\text { Nonspecific lymphadenopathy } \\
(\mathrm{n}=24)\end{array}$} & \multicolumn{2}{|c|}{$\begin{array}{l}\text { Other } \\
(\mathrm{n}=6)\end{array}$} & \\
\hline & $\mathrm{n}$ & $\%$ & $\mathbf{n}$ & $\%$ & $\mathrm{n}$ & $\%$ & $\mathbf{n}$ & $\%$ & $\mathbf{n}$ & $\%$ & \\
\hline \multicolumn{12}{|l|}{ Liver } \\
\hline Palpable & 15 & 51.7 & 3 & 9.4 & 9 & 37.5 & 5 & 20.8 & 0 & 0.0 & \multirow[t]{2}{*}{$0.001^{\mathrm{s}}$} \\
\hline Not palpable & 14 & 48.3 & 29 & 90.6 & 15 & 62.5 & 19 & 79.2 & 6 & 100.0 & \\
\hline \multicolumn{12}{|l|}{ Spleen } \\
\hline Palpable & 23 & 79.3 & 8 & 25.0 & 0 & 0.0 & 7 & 29.2 & 0 & 0.0 & \multirow[t]{2}{*}{$0.001^{\mathrm{s}}$} \\
\hline Not palpable & 6 & 20.7 & 24 & 75.0 & 24 & 100.0 & 17 & 70.8 & 6 & 100.0 & \\
\hline \multicolumn{12}{|l|}{ Ascites } \\
\hline Present & 2 & 6.9 & 2 & 6.3 & 9 & 37.5 & 2 & 8.3 & 0 & 0.0 & \multirow[t]{2}{*}{$0.002^{\mathrm{s}}$} \\
\hline Absent & 27 & 93.1 & 30 & 93.8 & 15 & 62.5 & 22 & 91.7 & 6 & 100.0 & \\
\hline
\end{tabular}

$\mathrm{s}=$ significant, $\mathbf{P}$ value reached from chi square test

Table Shows palpable liver was found $15(51.7 \%)$ in lymphoma, $9(37.5 \%)$ in metastatic carcinoma and 5(20.8\%) in nonspecific lymphadenopathy. Palpable spleen was found $23(79.3 \%)$ in lymphoma, $8(25.0 \%)$ in tuberculosis and $7(29.2 \%)$ in nonspecific lymphadenopathy. Ascites was found, $9(37.5 \%)$ in metastatic carcinoma $2(8.3 \%)$ in nonspecific lymphadenopathy $2(6.9 \%)$ in lymphoma, $2(6.3 \%)$ in tuberculosis and. Which were statistically significant $(<0.05)$ among five groups.

\section{DisCUSSION}

The present study tries to give an idea to proceed with such cases and also try to give insight to the medical professional about the overall quantum of the problem. Rahman et al., [11] designed a study in their population for histological evaluation of cervical lymph node biopsies that might be important in the management of these patients. Histopathological evaluation of different diseases involving the cervical lymph nodes in relation to age and sex of the study population. It was a cross sectional study conducted in the department of Pathology, a total of 107 patients were evaluated for specific cause of cervical lymphadenopathy in relation to age and sex. Lymph node biopsies of all patients of both sexes and all age groups were included in the study. Among the 107 subjects $58(54.2 \%)$ were males and $49(45.8 \%)$ were females with a male to female ratio of 1.2:1. The age of the patients ranged from 2 to 85 years with a mean age of $32.68 \pm 18.01$ years. Of the 107 lymph node biopsies, 34 cases $(31.8 \%)$ were reactive lymphadenitis, 41 cases $(38.3 \%)$ were tuberculosis, 2 cases $(1.9 \%)$ were noncaseous granuloma, 6 cases $(5.6 \%)$ were Hodgkin lymphoma, 8 cases $(7.5 \%)$ were non-Hodgkin lymphoma, 12 cases $(11.2 \%)$ were met static neoplasm and 4 cases $(3.7 \%)$ were other specific lesions. The commonest cause of cervical lymphadenopathy was tuberculosis, followed by reactive lymphadenitis, lymphoma and metastatic neoplasm. In our country a study done by Miah [1] to find out the variation in clinical presentation, aetiological pattern of Lymphadenopathy and to find out any variation of aetiological pattern of Lymphadenopathy in the northern part of Bangladesh in comparison to other parts of the country. Their observational study included 50 adult patients, admitted in medicine wards of Rangpur Medical college hospital. Aged >13 years, presented with generalized Lymphadenopathy or regional Lymphadenopathy and presented with enlarged lymph nodes $>2 \mathrm{~cm}$ in inguinal region and $>1 \mathrm{~cm}$ in the cervical and other regions were enrolled in their study. Aged under 13 years, extremely debilitated patient and patient with insignificant lymph node enlargement, e.g. $<1 \mathrm{~cm}$ in cervical regions and $<2 \mathrm{~cm}$ in inguinal regions were excluded from their study. Pandav et al., [12] described cytomorphological patterns of FNAC of cervical lymph nodes and its utility in establishing diagnosis. Biopsy and special stains were done in selected cases. Patients included in the present study were in the age group of 11 months to 80 years. Almost forty percent $(38.0 \%)$ cases were of tubercular lymphadenitis, $24.0 \%$ cases show Metastatic tumors, $22.0 \%$ hyperplasic lymph nodes, $11.0 \%$ acute lymphadenitis, $3.7 \%$ Lymphoma and $1.4 \%$ were Leukemia Lymphadenopathy. Tubercular lymphadenopathy was found with increasing frequency through adolescence $43.30 \%$ to young adulthood $54.75 \%$ \& $48.18 \%$ in adulthood. $30.59 \%$ cases of tuberculosis were in age group of 11-40 years. $17.8 \%$ cases of metastatic tumors were in the age group of 31 60 years. Highest incidence of metastatic malignancy was seen in the fifth decade $35.0 \%$. The most frequent causes of cervical lymphadenopathy are tuberculosis, metastatic malignancies and reactive lymphadenitis. 
Yaris et al., [13] study, the clinical and laboratory features of children with lymphadenopathy were evaluated. Over a 3-year period, 126 patients were referred to the clinic for lymphadenopathy. Twentyeight of cases have diseases mimicking lymphadenopathy; 98 (mean age: $86 \pm 55$ months) have lymphadenopathy. Localized, limited, and generalized involvement was found in $52 \%, 30 \%$, and $18 \%$ of patients. The most common localization was the head and neck region. The causes of lymphadenopathy were benign diseases in 75 patients. Sixty percent were reactive lymphadenopathy, 39\% were lymphadenitis. Lymphadenitis was more frequently localized and bigger than $3 \mathrm{~cm}$ compared with reactive adenopathy. Twenty-three patients have malignant diseases whose mean age was higher than others. The enlargement of supraclavicular nodes was more likely due to malignant disease. A total of 45 patients underwent FNAC in this study and it was observed that among them tuberculosis ranked on the top (46.7\%). In our country Biswas et al., [14] study showed that the tubercular lymphadenitis was $(45.4 \%)$, which is similar to our study. Another study done by Pandav et al., [12] found that $38.0 \%$ cases were of tubercular lymphadenitis which also consistent with our study. In our FNAC series next common case was lymphoma in $17.8 \%$ patients. which is consistent (about $17 \%$ of lymphoma cases) with study done by Ageep [15] but it is not consistent with study done by Biswas et al., [14] where lymphoma case is $7 \%$, which may be duo to common practice of biopsy in suspected case of lymphoma. Metastatic carcinoma $17.8 \%$, was next to lymphoma, this finding is similar to Biswas et al., [14] 21.2\% and also Pandav et al., [12] $24 \%$. Nonspecific lymphadenopathy in our series was $13.3 \%$ that is also similar with Biswas et al., [14] $19.9 \%$ but more in Pandev et al., [12] 22\%. It may be due to inclusion of only adult patient in our study. In this present series a total of 70 patient underwent lymph node Biopsy and it was observed that $30.0 \%$ patients had lymphoma, in our country Miah [1] showed lymphoma cases were (28\%), another study done in Saudi Arabia by Albasri et al., [16] which shows number of lymphoma were $24.6 \%$ which is similar to our study and according to Olu-Eddo \& Ohanaka [17] lymphoma cases were $23 \%$. In this series the second leading cause of lymphadenopathy were nonspecific lymphadenopathy (25.7\%), In India Mohan et al., [18] showed nonspecific lymphadenitis cases were $35.6 \%$. In our country Rahman et al., [11] observed nonspecific cases were $31.8 \%$ in his study. In both this study number of nonspecific cases were little bit higher than our study.

\section{Limitations OF THE STUdY}

The study population was selected from one selected hospital in Dhaka city, so that the results of the study may not be reflect the exact picture of the country.

\section{CONCLUSION AND RECOMMENDATIONS}

From this study, it is concluded that cervical lymphadenopathy is a common clinical problem and the commonest cause was tuberculosis followed by lymphoma and metastatic carcinoma. There were sufficient etiopathological and clinical features that differentiate age groups with lymphadenopathy. For evaluation of lymphadenopathy we should consider important signs and symptoms like "B" symptoms, consistency, fixity of lymph node and organomegaly. Lymph nodes were fixed in metastatic carcinoma and matted in case of tuberculosis. It will help the care giver doctor, especially at primary care level, to think in a systematic way for detection of respective cases early and thereby avoiding the diagnostic delay in cases like Tuberculosis and Malignancy. It can be of very helpful of the urban and rural primary health care levels, where in places improved diagnostic facility to detect lymph node diseases is yet to be available due to scarcity of resources. In cases of fever with cervical lymphadenopathy, fever is an important finding for think about common diseases like Tuberculosis, Lymphoma and metastatic diseases. Low grade fever is common in Tuberculosis and Lymphoma while high grade fever is commonly seen in metastatic diseases. FNAC may be a handy tool for metastatic disorder and tuberculosis whereas biopsy is almost certain for metastatic disorder or lymphoma. A good number of lymphadenopathy patients were nonspecific which need further investigation workup for confirmatory diagnosis. Lymph nodes are usually hard in metastatic carcinoma and matted in case of tuberculosis. Further studies can be undertaken by including large number of patients.

\section{REFERENCES}

1. Miah MEH. A study on clinical presentation and aetiological pattern of lymphadenopathy, BCPS dissertation. 2007; 37.

2. American Academy of Pediatrics. Tuberculosis. In: Pickering LK, ed. Red Book: 2003Report of the Committee on Infectious Diseases, 26th ed. Elk Grove Village, IL: American Academy of Pediatrics; 2003: 642-60.

3. Bazemore A, Smucker DR. Lymphadenopathy and malignancy. American family physician. $2002 \mathrm{Dec}$ 1;66(11):2103-2110.

4. Buchino JJ, Jones VF. Fine needle aspiration in the evaluation of children with lymphadenopathy. Archives of pediatrics \& adolescent medicine. 1994 Dec 1;148(12):1327-30.

5. Dajani AS, Garcia RE, Wolinsky E. Etiology of cervical lymphadenitis in children. New England Journal of Medicine. 1963 Jun 13;268(24):132933. 
6. Peters TR, Edwards KM. Cervical lymphadenopathy and adenitis. Pediatrics in review. 2000 Dec;21(12):399.

7. Spyridis P, Maltezou HC, Hantzakos A, Scondras C, Kafetzis DA. Mycobacterial cervical lymphadenitis in children: clinical and laboratory factors of importance for differential diagnosis. Scandinavian journal of infectious diseases. 2001 Jan 1;33(5):362-6.

8. Leung AK, Robson WL. Childhood cervical lymphadenopathy. Journal of Pediatric Health Care. 2004 Jan 1;18(1):3-7.

9. Ellison E, LaPuerta P, Martin SE. Supraclavicular masses: results of a series of 309 cases biopsied by fine needle aspiration. Head \& Neck: Journal for the Sciences and Specialties of the Head and Neck. 1999 May;21(3):239-46.

10. Zeharia A, Eidlitz-Markus T, Haimi-Cohen Y, Samra Z, Kaufman L, Amir J. Management of nontuberculous mycobacteria-induced cervical lymphadenitis with observation alone. The Pediatric infectious disease journal. 2008 Oct 1;27(10):920-2.

11. Rahman MA, Biswas MM, Siddika ST, Sikder AM. Histomorphological pattern of cervical lymphadenopathy. Journal of Enam Medical College. 2013 Feb 17;3(1):13-7.

12. Pandav AB, Patil PP, Lanjewar DN. Cervical lymphadenopathy-diagnosis by FNAC, a study of
219 cases. Asian J Med Res. 2012 Jun 15;1(3):7983.

13. Yaris N, Cakir M, Sözen E, Cobanoglu U. Analysis of children with peripheral lymphadenopathy. Clinical pediatrics. 2006 Jul;45(6):544-9.

14. Biswas G, Das A, Haldar D, Mukherjee A, Dutta S, Sinha R. Clinico-pathological correlates of cervical lymphadenopathy: a hospital based study. Indian Journal of Otolaryngology and Head \& Neck Surgery. 2013 Jul 1;65(1):42-7.

15. Ageep AK. Assessment of adult peripheral lymphadenopathy in Red Sea State, Sudan. Int J Trop Dis Health. 2012;2:24-32.

16. Albasri AM, El-Siddig AA, Hussainy AS, Alhujaily AS. Pattern of lymph node pathology in western Saudi Arabia. Asian Pac J Cancer Prev. 2014 Jan 1;15(11):4677-81.

17. Olu-Eddo AN, Ohanaka CE. Peripheral lymphadenopathy in Nigerian adults. JournalPakistan Medical Association. 2006 Sep;56(9):405-408.

18. Mohan A, Reddy MK, Phaneendra BV, Chandra A. Aetiology of peripheral lymphadenopathy in adults: analysis of 1724 cases seen at a tertiary care teaching hospital in southern India. National Medical Journal of India. 2007 Jan 1;20(2):78. 